\title{
Elastic and magnetic properties of the bilayer manganese oxide $\left(\operatorname{Pr}_{0.6} \mathrm{La}_{0.4}\right)_{1.2} \mathrm{Sr}_{1.8} \mathrm{Mn}_{2} \mathrm{O}_{7}$
}

\author{
Yoshiki Nakanishi,* Kota Shimomura, Tomoyuki Kumagai, Michiaki Matsukawa, and Masahito Yoshizawa \\ Graduate School of Frontier Materials Function Engineering, Iwate University, Morioka 020-8551, Japan \\ Ramanathaan Suryanarayanan ${ }^{\dagger}$ \\ Department of Physics and Astronomy, 666 West Hancock, Wayne State University, Detroit, Michigan 48201, USA
}

Jagdish Singh Thakur

Department of Electrical and Computer Engineering, 5050 Anthony Wayne Drive, Wayne State University, Detroit, Michigan 48202, USA

Mircea Apostu ${ }^{\ddagger}$ and Alexandre Revcolevschi

Laboratoire de Physico-Chimie de L'Etat Solide, UMR 8082, Université Paris-Sud, 91405 Orsay, France

Shintaro Nakamura

Center for Low-Temperature Science, Tohoku University, Sendai 980-8578, Japan

(Received 27 January 2007; revised manuscript received 8 June 2007; published 25 September 2007)

\begin{abstract}
The elastic and magnetic properties of a single crystal of the bilayer manganese oxide $\left(\mathrm{Pr}_{0.6} \mathrm{La}_{0.4}\right)_{1.2} \mathrm{Sr}_{1.8} \mathrm{Mn}_{2} \mathrm{O}_{7}$ have been investigated by means of ultrasonic and high-field magnetization measurements. Remarkable changes in the elastic constants $C_{11}, C_{33}, C_{44}$, and $C_{66}$ as a function of temperature and magnetic field have been observed. In particular, a distinct elastic anomaly was observed at low temperatures and in magnetic fields when crossing the phase boundary between the paramagnetic insulating and the fieldinduced ferromagnetic metallic state. A pronounced elastic softening as a function of magnetic field $(H)$ appears across the boundary of the low-temperature magnetic phase below around $40 \mathrm{~K}$, accompanied by a distinct hysteresis. In the high-field region, however, these elastic constants exhibit a monotonic increase upon increasing the magnetic field. The high-field magnetization measurements characterizing the magnetic state point out a strong coupling between the elastic strain and the magnetic moment. The data can be described reasonably well considering a strong coupling between elastic strain and magnetic susceptibility $\chi_{m}=\partial M / \partial H$.
\end{abstract}

DOI: 10.1103/PhysRevB.76.094416

PACS number(s): 71.27. $+\mathrm{a}, 71.30 .+\mathrm{h}, 75.30 . \mathrm{Kz}$

\section{INTRODUCTION}

Manganites exhibit interesting original properties such as colossal magnetoresistance (CMR), giant magnetostriction, etc., which are thought to originate from a competition between charge, lattice, and spin degrees of freedom. Even though the fundamental mechanism of CMR is reasonably accounted for within the framework of the double exchange arising from the hopping of mobile $e_{g}$ electrons between $\mathrm{Mn}^{3+}$ and $\mathrm{Mn}^{4+}$ ions, ${ }^{1,2}$ several other properties such as insulating ferromagnetic ground state, giant magnetostriction, magneto-thermal conductivity, etc., are not well understood. Among manganites, the double layer compound $\mathrm{La}_{2-2 x} \mathrm{Sr}_{1+2 x} \mathrm{Mn}_{2} \mathrm{O}_{7}$ has attracted considerable attention due to the enhanced CMR effect observed for $x=0.4$ and attributed normally to its quasi-two-dimensional structure, ${ }^{3,4}$ made of two $\mathrm{MnO}_{6}$ layers alternately stacked with (La, $\left.\mathrm{Sr}\right) \mathrm{O}_{2}$ layers along the $c$ axis of the structure. Furthermore, it was shown that substitution of $\mathrm{Pr}^{3+}$ for $\mathrm{La}^{3+}$ at the concentration of $z=0.6$ in $\left(\mathrm{La}_{1-z} \mathrm{Pr}_{z}\right)_{1.2} \mathrm{Sr}_{1.8} \mathrm{Mn}_{2} \mathrm{O}_{7}$ (hereafter denoted as PLSMO) resulted in a small contraction of the $a$ parameter from $a=3.875 \AA$ to $a=3.863 \AA$ and an expansion of the $c$ parameter from $c=20.13 \AA$ to $c=20.15 \AA$. This gave rise to unexpected interesting properties. The ferromagnetic transition at $T_{c}=125 \mathrm{~K}$, accompanied by an insulator to metal transition observed in the compound with $z(\operatorname{Pr})=0$, was completely suppressed in the compound with $z(\operatorname{Pr})=0.6$. However, a magnetic field could induce a field-induced first-order paramagnetic insulator to ferromagnetic metal transition in this latter compound. In addition, a huge decrease of the $c$-axis resistivity by a factor of $1 \times 10^{6}$ was observed at $T$ $=5 \mathrm{~K}$ in the presence of a magnetic field of $5 \mathrm{~T} .{ }^{5,6}$ The firstorder transition was also accompanied by a remarkable negative magnetoresistance when the field was oriented along the $c$ axis, indicating the presence of an important interplay between spin, carrier, and orbital degrees of freedom. ${ }^{7,8}$ A polarized neutron study of this compound revealed an increase in the population of the $d\left(3 z^{2}-r^{2}\right)$ orbitals of $\mathrm{Mn}^{3+}$ in the field-induced ferromagnetic state when the field was along the $c$ axis. ${ }^{9,10}$ In order to investigate further this interplay, we have studied the elastic properties of this compound as a function of temperature and magnetic field. Ultrasonic sound velocity measurements were used to determine the elastic constants. We expected indeed that near such a field-induced phase transition, the elastic strain would couple to the fluctuation which, in the case of magnetic transitions, would produce noticeable changes in the sound velocity that would imply changes in the elastic constants. A preliminary report of our first results has been published in Ref. 11.

\section{EXPERIMENT}

The single crystal of PLSMO used for our experiments was grown by the floating-zone method using a mirror furnace and cut to a size of $5 \times 4 \mathrm{~mm}^{2}$ in the $a b$ plane and 
$1 \mathrm{~mm}$ along the $c$ axis. ${ }^{5}$ The sound velocity $(v)$ was measured by the pulse echo method with carrier frequencies of 5 and $10 \mathrm{MHz}$ for longitudinal and transverse waves, respectively. For precise measurement of the sound velocity, the constant phase method was introduced. The echo signals were multiplied by the in phase and quadrature phase references using a double-balanced mixer (DBM), and the two outputs of DBMs through a low pass filter were detected independently. The frequency was controlled to maintain a constant phase between the two outputs. The sound velocity change is thus obtained from the frequency change (phase comparison method). The resolution in the relative velocity was $10^{-6}$ in the present work. Plates of $\mathrm{LiNbO}_{3}$ transducers were used for the generation and detection of the sound waves. The transducers were glued on the parallel faces of the sample by the elastic polymer Thiokol. We have performed, in addition, high-field magnetization $(M)$ measurements with the help of a superconducting quantum interference device magnetometer operating between 1.8 and $300 \mathrm{~K}$ in magnetic fields up to $5 \mathrm{~T}$ and a vibrating sample magnetometer in magnetic fields up to $12 \mathrm{~T}$ and at temperatures down to $4.2 \mathrm{~K}$.

\section{EXPERIMENTAL RESULTS}

\section{A. Magnetization measurements}

In this section, we briefly recall the magnetic properties of our sample and present a few additional measurements. In an earlier study, we have reported a cusp in the zero-fieldcooled (ZFC)-field cooled curve around $40 \mathrm{~K}$, in fields up to $5 \mathrm{~T}$, that can be attributed to a spin-glass-like behavior. ${ }^{5} \mathrm{Sig}-$ nificant differences were observed in the resistivity when it was measured along the $c$ axis or in the $a b$ plane, and the magnetization data revealed that the $c$ axis was the easy axis of the magnetization. Figure 1 shows the magnetization of our sample at several temperatures ranging from 10 to $200 \mathrm{~K}$ as a function of magnetic field applied along the $c$ axis. The data clearly indicate that, for $T<100 \mathrm{~K}$, the magnetization shows an increase for $H>4 \mathrm{~T}$, along the $c$ axis. A sharp transition with a pronounced hysteresis is clearly visible at $T=3.7 \mathrm{~K}$ for a critical field of about $5 \mathrm{~T}$, as shown in Fig. 2 . These data clearly indicate a metamagnetic transition to a field-induced ferromagnetic state. The transition is observed at higher fields and becomes smeared at higher temperatures. It can also be noted that the transition is no longer accompanied by hysteresis above $50 \mathrm{~K}$. Furthermore, the metamagnetic transition was undetectable at $200 \mathrm{~K}$.

\section{B. Ultrasonic measurements}

In this section, we report the elastic property measurements of PLSMO. Figure 3 shows the temperature dependence of the relative changes in the longitudinal elastic constants $C_{11}$ and $C_{33}$ and the transverse elastic constants $C_{44}$ and $C_{66}$ in zero field. In order to avoid overlap and for clarity, the curves are shifted arbitrarily. We recall that $C_{11}$ and $C_{33}$ are the elastic constants determined by a longitudinal sound wave propagating along the $a$ and $c$ axes, respectively, and $C_{44}$ and $C_{66}$ are those determined by a transverse one

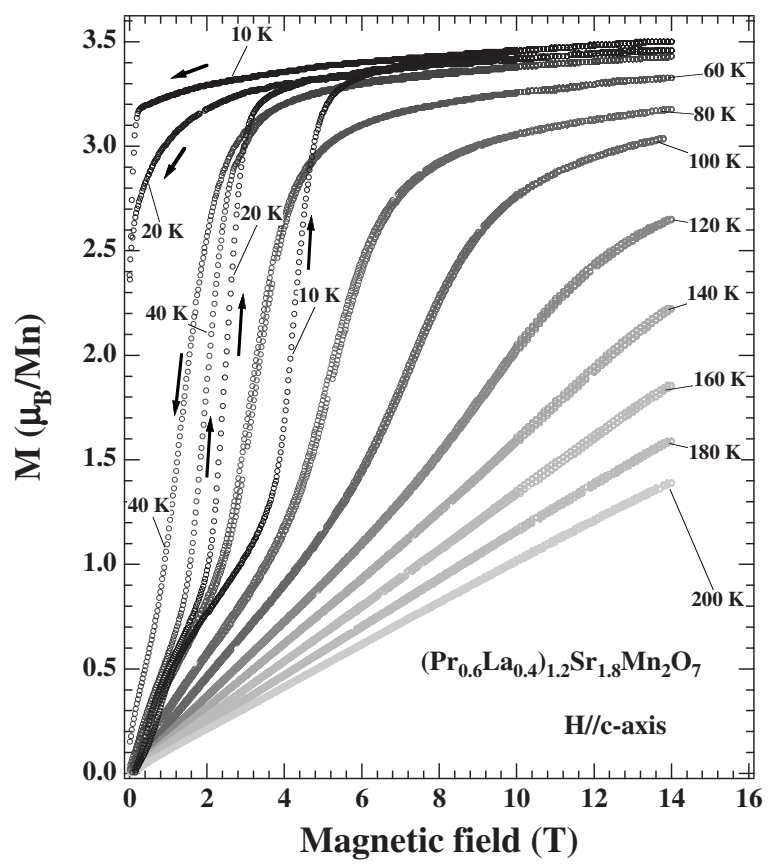

FIG. 1. Field dependence of the magnetization for a field applied along the $c$ axis above $10 \mathrm{~K}$.

propagating along the $a$ axis with polarization along the $c$ axes and propagating along the $c$ axes with polarization along the $a$ axes, respectively. Both increase monotonically with decreasing temperature. A slight anomaly is observed around $T^{*}=40 \mathrm{~K}$ and coincides with the earlier reported cusp in the magnetization data in low fields. However, a pronounced elastic anomaly was induced by application of magnetic fields. Figure 4 shows the field dependence of $\Delta C_{33} / C_{33}$ at selected temperatures. At $4.2 \mathrm{~K}$, the value of $\Delta C_{33} / C_{33}$ decreases sharply at $6 \mathrm{~T}$, and this is followed by a small increase for a further increase of the field. A strong

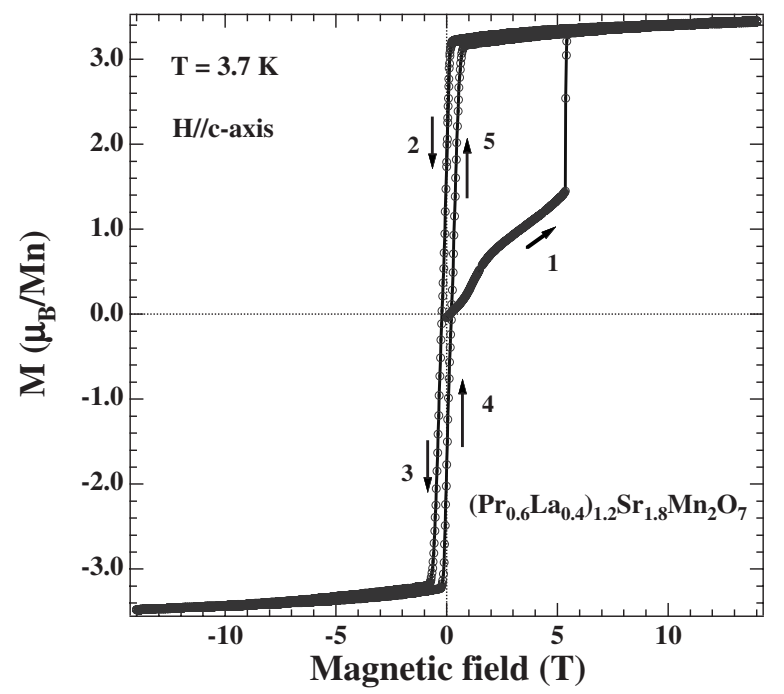

FIG. 2. Field dependence of the ZFC magnetization for a field applied along the $c$ axis at $3.7 \mathrm{~K}$. The label against each curve indicates the order of the magnetic field. 


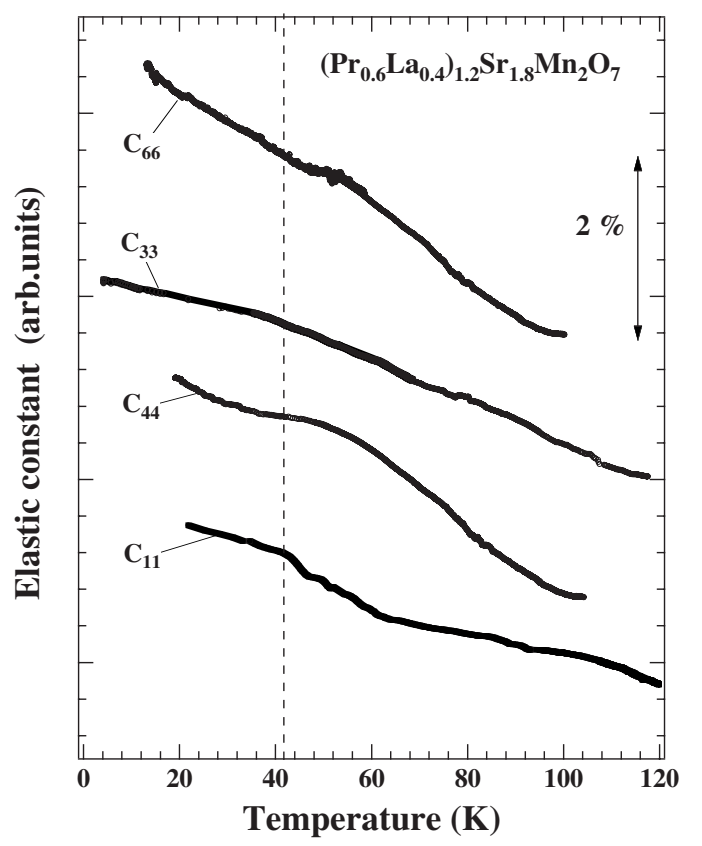

FIG. 3. Temperature dependence of elastic constants $C_{11}, C_{33}$, $C_{44}$, and $C_{66}$ of LPSMO for $z=0.6$ in the absence of magnetic field. The dotted vertical line indicates the transition point determined by the resistivity measurements.

hysteresis, quite similar to that of the magnetic transition and indicative of a first-order transition, was observed when the field was decreased from $10 \mathrm{~T}$ to zero. The hysteresis was still observed for $T=30 \mathrm{~K}$. However, at $T=55 \mathrm{~K}$, no more

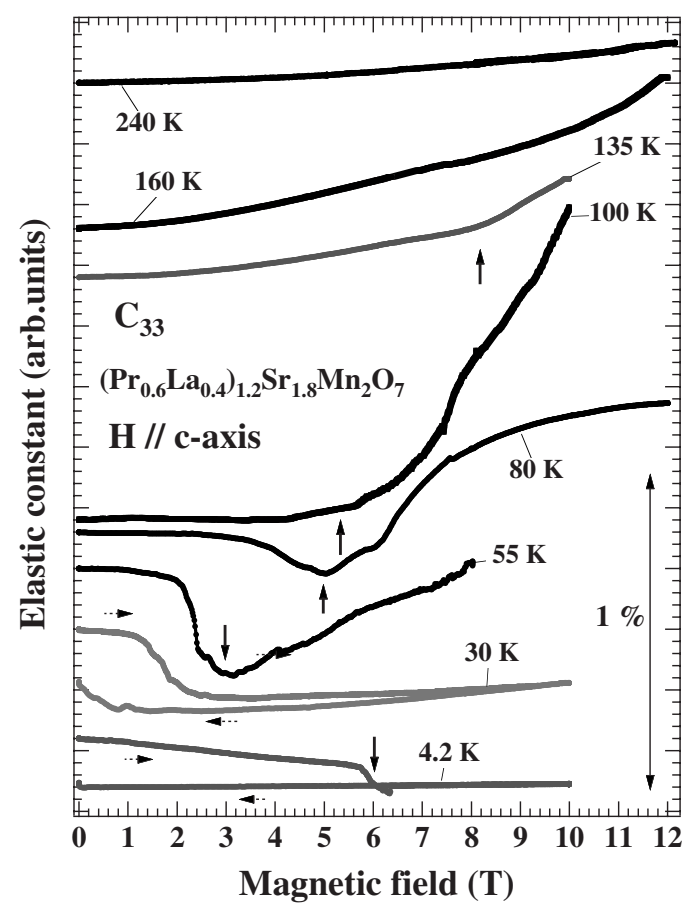

FIG. 4. Magnetic field dependence of the elastic constant $C_{33}$ of PLSMO at selected temperatures. The vertical arrows indicate a transition point. The horizontal ones indicate the direction of the applied fields.

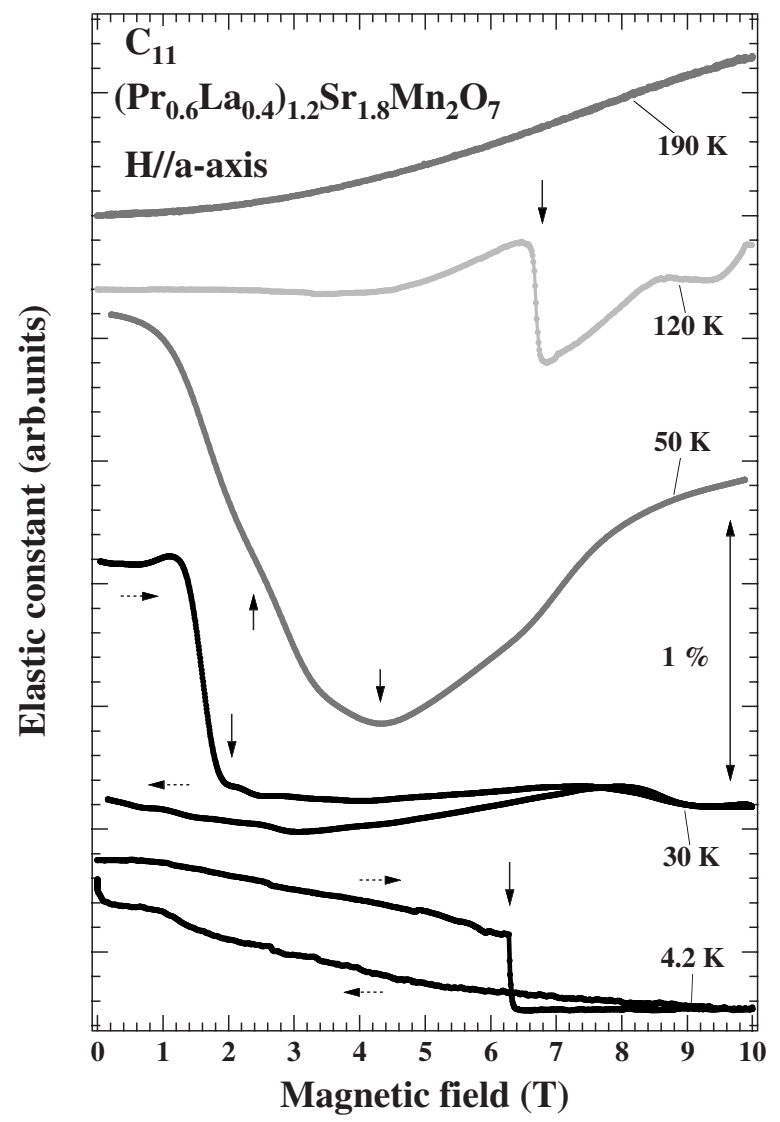

FIG. 5. Magnetic field dependence of the elastic constant $C_{11}$ of PLSMO at selected temperatures. The vertical arrows indicate a transition point. The horizontal ones indicate the direction of the applied fields.

hysteresis was observed and the value of $\Delta C_{33} / C_{33}$ increased abruptly for $H<3 \mathrm{~T}$. This increase was observed for $T$ $<135 \mathrm{~K}$. Figure 5 shows the field dependence of $\Delta C_{11} / C_{11}$ at selected temperatures. Although the general behavior was similar to that of $\Delta C_{33} / C_{33}$, additional anomalies were observed at $120 \mathrm{~K}$ and at $160 \mathrm{~K}$. Figures 6 and 7 show the field dependence of $\Delta C_{44} / C_{44}$ and of $\Delta C_{66} / C_{66}$ at selected temperatures, respectively. The distinct elastic anomalies are also observed in the transverse elastic constant measurements. It is noted that $C_{44}$ exhibits a sharp jump at the transition field, at $4.2 \mathrm{~K}$, unlike $C_{11}$ and $C_{33}$. Hysteresis effects are also observed. If we exclude these effects, we can say that the whole behavior around the transition field and also the elastic constants are qualitatively similar to those of longitudinal $C_{11}$ and $C_{33}$.

\section{Magnetic phase diagram}

From these elastic and magnetic data, we can construct an $(H-T)$ phase diagram for PLSMO, shown in Fig. 8. The transition points were determined by the inflection points at the $M-H$ curves and $H-T$ ones reported previously by our group. It is inferred from the observed features that there are, at least, three different phases I, II, and (III, III'). Regions I and II represent paramagnetic and field-induced ferromagnetic 


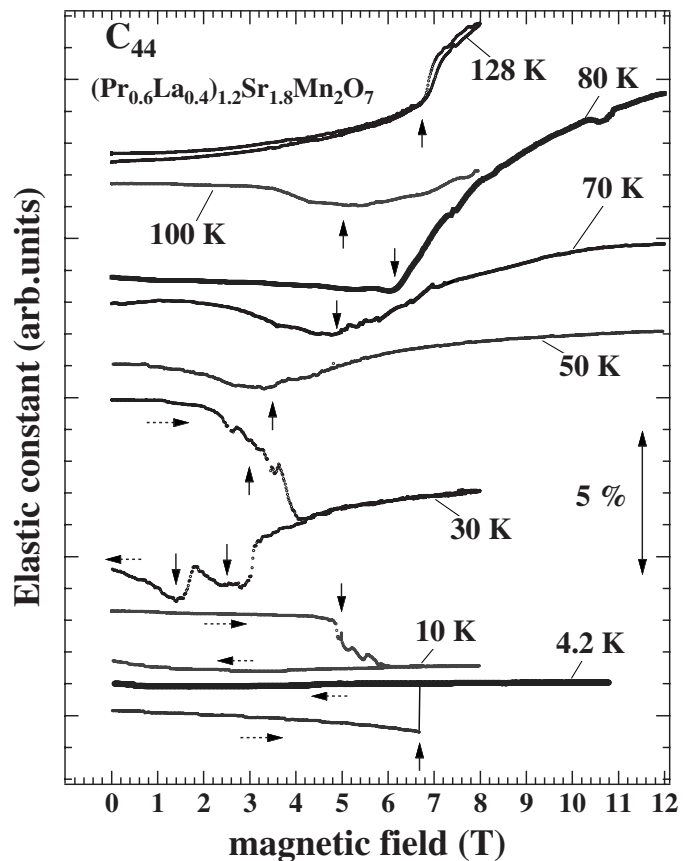

FIG. 6. Magnetic field dependence of elastic constant $C_{44}$ of PLSMO at selected temperatures. The vertical arrows indicate a transition point. The horizontal ones indicate the direction of the applied fields.

phases, respectively. An almost full magnetic moment of $3.0 \mu_{B} / \mathrm{Mn}$ is induced in phase II, where the system exhibits a metallic behavior of the resistivity. Thus, phase II is fully field-induced ferromagnetic phase. On the other hand, phase III is also considered to be a partial field-induced ferromag-

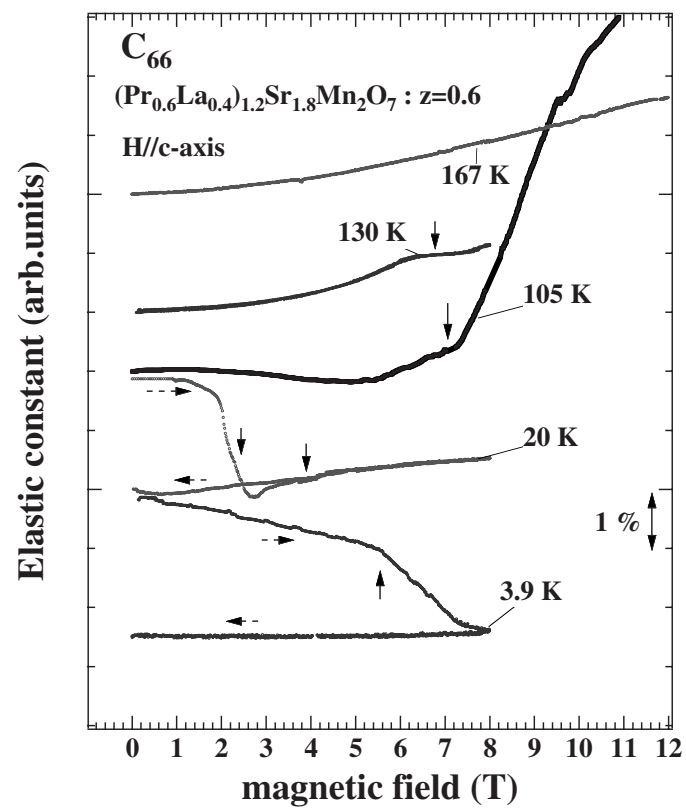

FIG. 7. Magnetic field dependence of elastic constant $C_{66}$ of PLSMO at selected temperatures. The vertical arrows indicate a transition point. The horizontal ones indicate the direction of the applied fields.

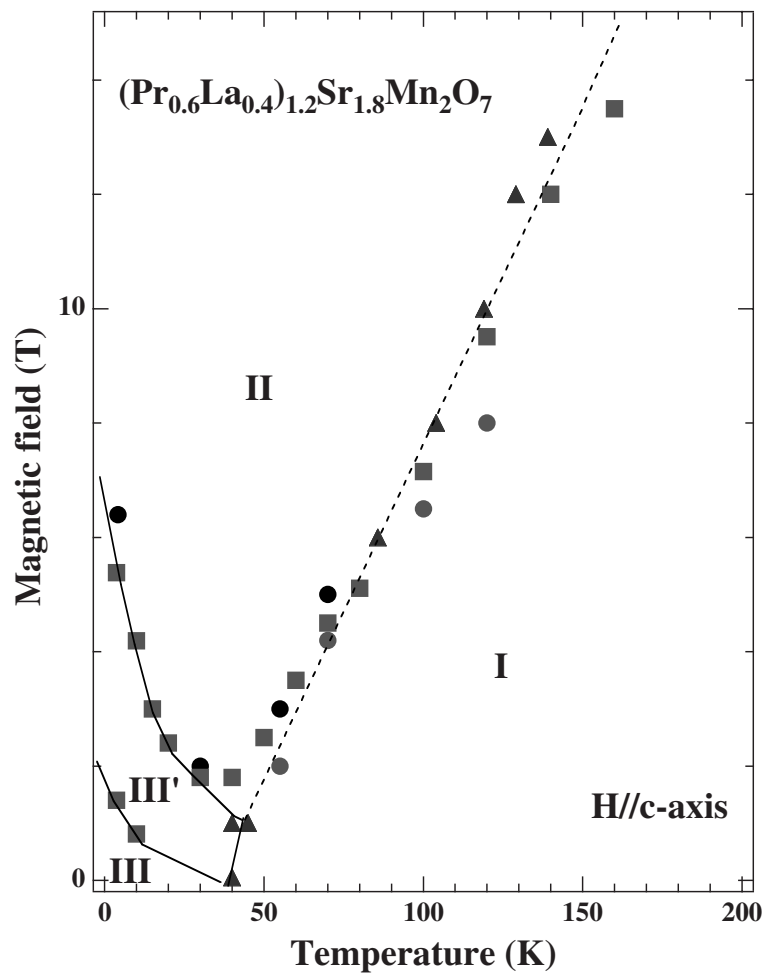

FIG. 8. (H-T) magnetic phase diagram determined by the present results. The squares, triangles, and circles denote the transition points determined by the $M-H$ curves, $M-T$ curves, and elastic constant measurement, respectively. The solid and dotted lines are guides to the eye. The solid and dotted lines denote a phase boundary with or without hysteresis effect, respectively.

netic phase. There are two subregions in phase III where the boundary is defined only by the anomaly in the virgin $M-H$ and $C-H$ curves. It seems that this boundary may be due to domain rotation. It is noted that the boundary between I and III is poorly defined in zero field, whereas it becomes distinct, gradually with increasing field, indicating that phase III appears only under magnetic field. The transition is accompanied by a remarkable hysteresis in both magnetization and elastic constants at the boundary between phases III and II, indicating a first-order transition. In contrast, no hysteresis is observed between phases I and II, and given that the boundary between phases I and II ends at around $150 \mathrm{~K}$, the transition is a first-order type. Although there is an insulatormetal transition on crossing the phase boundary between phases I and II, the symmetry of the system remains unchanged. Only on the zero magnetic field axis of the phase diagram there is a second-order phase transition, but that is between phases I and III. The qualitative features of the elastic constants can be summarized as follows. The elastic constant decreases when crossing the phase boundary from phase III to phase II, and a remarkable hysteresis is observed only in the virgin run upon increasing the field. However, it decreases gradually with increasing field in phase I and increases gradually after crossing the boundary from phase I to phase II, without any hysteresis. The temperature of $T^{*}$ $=40 \mathrm{~K}$ is likely to be the critical temperature distinguishing these phases, although $T^{*}$ is much less distinct in zero field. 
Strong short-range-ordered spin correlations may arise around $T^{*}$. However, it seems that field application causes the stabilization of ferromagnetic long-range order, as will be discussed in detail below.

\section{DISCUSSION}

The major findings of this work are the observation of the strong magnetic field dependence of the elastic constants $C_{i i}$ for both longitudinal and transverse waves. This shows that the ordering of the magnetic moments clearly affects the elastic properties of the magnetic materials. A sudden transition is also observed in the longitudinal elastic constant $C_{11}$ and in the transverse elastic constant $C_{44}$ for field values around $7 \mathrm{~T}$, at $4.2 \mathrm{~K}$. Another interesting feature of these constants is the observation of an hysteresis as a function of applied magnetic field for temperatures less than or equal to $40 \mathrm{~K}$. The magnetic field value at which the hysteresis occurs depends on the elastic constant and temperature. However, the magnetic moments $M_{c}$ and $M_{a b}$ did not show any transition as a function of applied magnetic field where hysteresis or a sudden softening of the elastic constants was observed. This could be due to the very different time scales of the excitation of the lattice and magnetic moments of the systems. In general, the elastic constants vary with magnetic field, suggesting appreciable interaction between magnetic field and lattice. It is worthwhile discussing the behavior of the magnetic susceptibility $\chi_{m}=\partial M / \partial H$, a quantity which is intimately related to a change in the value of the lattice constant with magnetic field, according to Refs. 12 and 13. For heavy fermion systems, it has been observed that the magnetic susceptibility $\chi_{m}=\partial M / \partial H$ is directly related to the softening of the longitudinal modes according to the relation

$$
\Delta C_{\Gamma}=-\Gamma_{B}^{2} H^{2} \chi_{m},
$$

where $\Gamma_{B}=-\partial \ln H c / \partial \varepsilon_{\Gamma}$ is the Grüneisen parameter and $\varepsilon_{\Gamma}$ is the elastic strain with $\Gamma$ symmetry. We verify this relation by estimating $\Gamma_{B}^{2}$ from the plots of $\chi_{m} H^{2}$ and $\Delta C_{33} / C_{33}$ as a function of magnetic field as shown in Figs. 9 and 10, respectively, for different values of temperature. We find that the values of $\Gamma_{B}^{2}$ calculated at the critical field is almost constant or varies over a very small range for all the temperatures. Although some of their quantitative aspects do not agree well between the experimental results and the calculated ones, we emphasize that this agreement gives evidence for a strong spin-lattice coupling in PLSMO above $T^{*}$ $=40 \mathrm{~K}$. The expected upturn of the elastic constants by $-\chi_{m} H^{2}$ above $H_{c}$, below $40 \mathrm{~K}$, does not appear in the measured elastic constants, which is expected from Eq. (1). One scenario to explain the discrepancies is that they result from a change of the carrier number in the field-induced metallic phase, as will be discussed in detail below.

Obviously, the physics of these materials is dominated by the double-exchange process, electron-phonon coupling, and the electron kinetic energy represented by the bandwidth of the $e_{g}$ band. The hopping of the $e_{g}$ electrons, which promotes the ferromagnetic order, is affected by the values of the lattice constants in addition to the relative alignment of the core spins. The Jahn-Teller effect involves the simultaneous split-
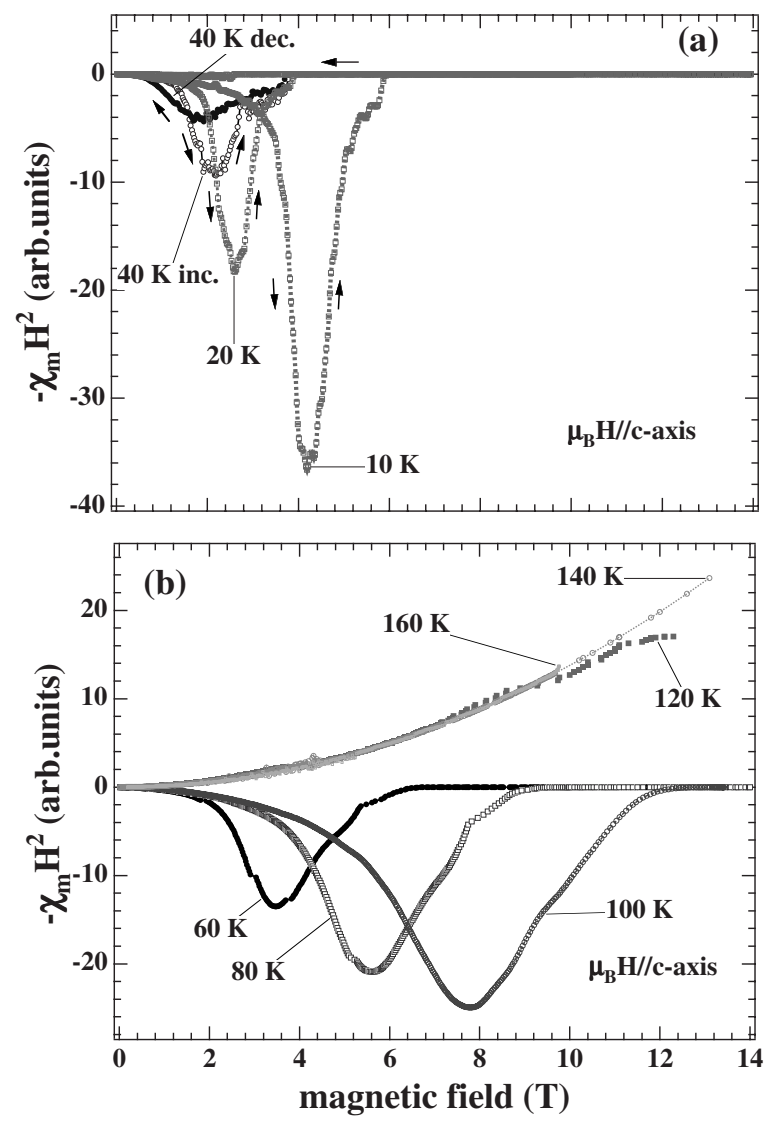

FIG. 9. Field dependence of $-H^{2} \chi_{m}$ (a) below the critical temperature of $40 \mathrm{~K}$ and (b) above $40 \mathrm{~K}$. The dotted arrows indicate the direction of the applied fields. $40 \mathrm{~K}$ inc. and $40 \mathrm{~K}$ dec. denote a result at $40 \mathrm{~K}$ in the increase and decrease processes of the magnetic field, respectively.

ting of the electronic states and the symmetry lowering distortion of the lattice. The distortion of the $\mathrm{MnO}_{6}$ octahedra couples the magnetic and lattice vibrations, resulting in the fact that electronic, lattice, and magnetic degrees of freedom are intimately connected. This affects both the exchange interaction and the crystal field potential. The coupling changes the single particle electron state energy $E_{k}^{0}$, and, according to the deformation potential coupling theory, the modified energy is given by

$$
E_{k}(\varepsilon)=E_{k}^{0}+d_{\Gamma}(k) \varepsilon_{\Gamma},
$$

where $d_{\Gamma}$ is the deformation potential coupling constant with $\Gamma$ symmetry. ${ }^{14-17}$ The index $\Gamma$ denotes the irreducible representation in the tetragonal symmetry. ${ }^{18}$ The free energy $F_{e l}$ for the mobile electrons can be written as

$$
F_{e l}=n E_{F}-k_{B} T \sum_{k} \ln \left(1+\exp \left(\frac{\left(E_{F}-E_{k}\right)}{k_{B} T}\right)\right) .
$$

Here, $E_{F}$ and $n$ denote the Fermi energy and the number of the mobile electrons in the $e_{g}$ band, respectively.

Using Eqs. (2) and (3), the elastic constant $C_{\Gamma}$ $=\partial^{2} F_{e l} / \partial \varepsilon \Gamma^{2}$ for a wave vector $k$-dependent deformation potential coupling constant can be written as 


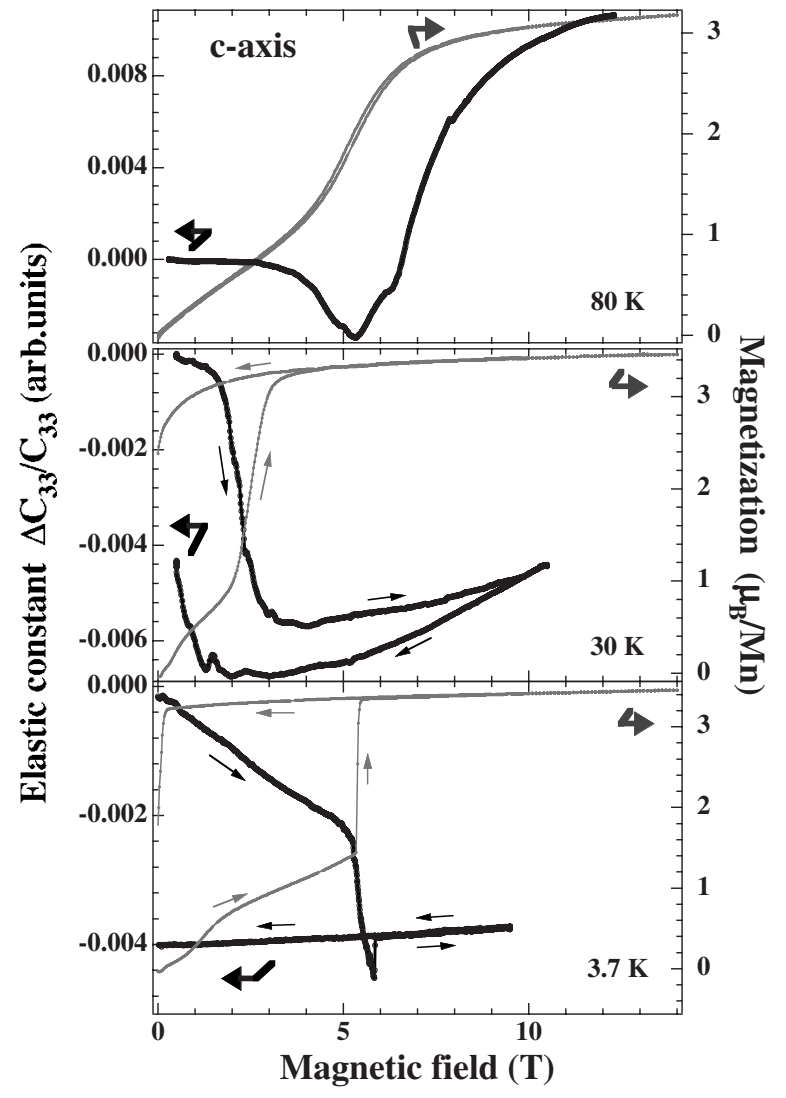

FIG. 10. Comparison of the obtained elastic constants with the magnetization at $3.7,30$, and $80 \mathrm{~K}$, respectively.

$$
\begin{aligned}
\Delta C= & -\frac{1}{k_{B} T} \sum_{k} d_{\Gamma}(k)^{2} f_{k}\left(1-f_{k}\right) \\
& +\frac{1}{k_{B} T}\left(\sum_{k} d_{\Gamma}(k)^{2} f_{k}\left(1-f_{k}\right)\right)^{2} / \sum_{k} f_{k}\left(1-f_{k}\right),
\end{aligned}
$$

where $\Delta C_{\Gamma}=C_{\Gamma}-C_{\Gamma}^{0}$ and $C_{\Gamma}^{0}$ is the elastic constant without magnetic field. In the expression of $\partial^{2} F_{e l} / \partial \varepsilon_{\Gamma^{2}}$, the $\Sigma_{k}\left(\partial F_{e l} / \partial \varepsilon_{\Gamma}\right)^{2} f_{k}$ term is zero due to the linear dependence of the dispersion $E_{k}(\varepsilon)=E_{k}^{0}+d_{\Gamma}(k) \varepsilon_{\Gamma}$ on $\varepsilon_{\Gamma}$. For a single band system, no changes in the elastic constants will be observed for a $k$-independent $d_{\Gamma}(k)$. However, for a two-band model, if $d_{\Gamma}(k)$ is independent of $k$ [i.e., $\left.d_{\Gamma}(k)=d_{\Gamma}\right], \Delta C_{\Gamma}$ is nonzero only if each band has a different value of $d_{\Gamma}$. From the general equation (4), it is clear that for a dispersive $d_{\Gamma}(k)$, which is the case with $\left(\operatorname{Pr}_{0.6} \mathrm{La}_{0.4}\right)_{1.2} \mathrm{Sr}_{1.8} \mathrm{Mn}_{2} \mathrm{O}_{7}$, the sudden changes in the elastic constants arise from the occupation probability functions which are affected when $d$ band splits into two bands due to the Jahn-Teller interaction. The electronphonon coupling originates from the Jahn-Teller splitting of the $d$ levels of the Mn ions whose electronically active orbitals are $d\left(x^{2}-y^{2}\right)$ and $d_{2}\left(3 z^{2}-r^{2}\right)$, and we assume that these orbitals form a two-band model leading to the "band-JahnTeller effect." A sudden change in the occupancy level of electrons in these bands upon application of a magnetic field could lead to a sudden change in the values of the elastic constants. The resistivity of these materials did not show any sudden transition ${ }^{19}$ with magnetic field, suggesting that the sudden transition in the elastic constants may not be the result of $e_{g}$ electron interaction with the lattice. The decrease of the resistivity upon increasing the magnetic field leads to the decrease of the elastic constant values as described by Eq. (4). It is inferred that this may suppress the upturn in the field dependence of the elastic constant just above $H_{c}$, expected from Eq. (1), as shown in Fig. 9. Unfortunately, we cannot discuss these changes quantitatively at present since the absolute values of the elastic constants were not determined. Nevertheless, we suggest that the elastic anomaly and the absence of the upturn of the elastic constant above $H_{c}$, below $T^{*}$, are ascribable to the coupling effect between the relevant elastic strain and the magnetic moment and between the elastic strain and the mobile $\mathrm{Mn} e_{g}$ electrons.

Next, we would like to comment on the low-temperature and low-field magnetic phase III, shown in Fig. 8. The characteristic features observed may suggest that this phase is fairly metastable. Figures 4-6 give typical examples to describe this situation. Actually, oscillatory phenomena are observed for $C_{33}$ at $30 \mathrm{~K}, C_{11}$ at 30 and $50 \mathrm{~K}$, and $C_{44}$ at 10 and $30 \mathrm{~K}$, below $H_{c}$. The oscillations disappear above $H_{c}$ and at higher temperatures. We conjecture that these oscillations can be related to the vicinity of a magnetic instability, such as a spin-glass state, since the transition is accompanied by a prominent hysteresis in both the ultrasonic and magnetization measurements. However, this is somehow unusual, because a slight anomaly is observed in zero field. The spinglass-like behavior shows up only by applying magnetic field. The critical temperature in zero field seems to be around $T^{*}=40 \mathrm{~K}$ by interpolation of the boundary between phases I and III if we assume that the transition line continues linearly with magnetic field. A slight anomaly was observed in both the temperature dependence of the elastic constant and magnetization curve at $T^{*}$. It is interesting to compare the magnetic properties of the compounds $\left(\mathrm{La}_{1-z} \mathrm{Nd}_{z}\right)_{1.2} \mathrm{Sr}_{1.8} \mathrm{Mn}_{2} \mathrm{O}_{7}$ and $\left(\mathrm{La}_{0.8} \mathrm{Gd}_{0.2}\right)_{1.4} \mathrm{Sr}_{1.6} \mathrm{Mn}_{2} \mathrm{O}_{7}$, reported previously by Moritomo et al. ${ }^{20}$ and Dho et al., ${ }^{21}$ respectively. The prominent features of their phase diagrams correspond to those obtained in $\left(\mathrm{La}_{1-z} \mathrm{Nd}_{z}\right)_{1.2} \mathrm{Sr}_{1.8} \mathrm{Mn}_{2} \mathrm{O}_{7}(z$ $=0.4$ ) at low temperature. In comparison, phase III is presumably due to a metastable region where the parainsulator and ferrometal states can coexist. That is to say ferromagnetic clusters can be grown by application of a magnetic field. This phase was understood within the framework of spin degree of freedom, so far. Our previous results for PLSMO have indicated that the magnetostriction (MS) along the $c$ axis and in the $a b$ plane showed a sudden decrease and increase at the transition field, respectively. ${ }^{7,8}$ The formation of domains due to bound carriers is also suggested by recent optical conductivity measurements on the compound $\left(\mathrm{Pr}_{0.6} \mathrm{La}_{0.4}\right)_{1.2} \mathrm{Sr}_{1.8} \mathrm{Mn}_{2} \mathrm{O}_{7}{ }^{22}$ The measurements did not show any metallic behavior even for magnetic fields up to $10 \mathrm{~T}$ in the ferromagnetic metallic state, but, instead, a far-infrared bound carrier excitation was inferred.

Furthermore, it should be noted that the transition is accompanied by a prominent hysteresis in MS. This implies that an orbital degree of freedom plays a crucial role in this ordered phase III as well. The inflection points in the elastic constant for field-increasing and field-decreasing runs shift 
toward low fields with increasing temperature in phase III, leading to a reduction of the field at which hysteresis sets in. Classical thermodynamics indicates that the first-order phase transition of the metastable state can occur in the condition that the potential barrier energy between two states becomes comparable with the temperature. ${ }^{20}$ Thus, it becomes difficult to shift from the metastable state to the other upon decreasing the temperature. This energy difference may correspond to $T^{*}=40 \mathrm{~K}=3.3 \mathrm{meV}$. The data relative to the elastic constants and MS as a function of field imply that the paramagnetic and ferromagnetic states are different. As was seen in $\mathrm{La}_{1.2} \mathrm{Sr}_{1.8} \mathrm{Mn}_{2} \mathrm{O}_{7}$, the $e_{g}$ state is dominated by $d\left(x^{2}-y^{2}\right)$ in the ferromagnetic phase, whereas some of the $d\left(3 z^{2}-r^{2}\right)$ population mixes with the $d\left(x^{2}-y^{2}\right)$ component in the paramagnetic phase. Recently, this behavior was confirmed microscopically by neutron scattering measurements. ${ }^{23,24}$ It seems that a spatial disorder of the orbital state may arise as well, in addition to this magnetic disorder in this system.

\section{CONCLUDING REMARKS}

We have performed magnetization and ultrasonic measurements on a single crystal of $\left(\mathrm{Pr}_{0.6} \mathrm{La}_{0.4}\right)_{1.2} \mathrm{Sr}_{1.8} \mathrm{Mn}_{2} \mathrm{O}_{7}$ as a function of temperature and magnetic field. A pronounced elastic anomaly was observed when crossing the boundaries of the phase diagram. In the absence of a microscopic theoretical descriptions, we proposed a phenomenological interpretation of our data in terms of the coupling between elastic strain and $\chi_{m}=\partial M / \partial H$. Various phases in the $(H-T)$ magnetic phase diagram of this system were discussed, and we observed a field-induced magnetic phase which exhibited hysteresis. Characteristic features of this phase suggest a strong coupling between the spin, orbital, and lattice of Mn ions degrees of freedom. It is noteworthy that the remarkable hysteresis in the elastic constants and also in magnetostriction was observed in the vicinity of the boundary between phases III' and II.

\section{ACKNOWLEDGMENTS}

We are grateful to Y. Tobinai, H. Uematsu, and M. Nakamura for their help in the ultrasonic measurements and the operation of the cryogenic apparatus. The measurements were performed in the Cryogenic Division of the Center for Instrumental Analysis, Iwate University. This work was partly supported by a Grant-in-Aid for Science Research from the Minister of Education, Culture, Sports, Science, and Technology of Japan. *yoshiki@iwate-u.ac.jp

†Permanent address: Laboratoire de Physico-Chimie de L'Etat Solide, UMR 8082 Université Paris-Sud, 91405 Orsay, France.

Permanent address: Faculty of Chemistry, Physical, Theoretical and Materials Chemistry, Al. I. Cuza University, Carol I, Iasi 700506, Romania.

${ }^{1}$ C. Zener, Phys. Rev. 82, 403 (1951).

${ }^{2}$ P. W. Anderson and H. Hasegawa, Phys. Rev. 100, 675 (1955).

${ }^{3}$ Y. Moritomo, A. Asamitsu, H. Kuwahara, and Y. Tokura, Nature (London) 380, 141 (1996).

${ }^{4}$ T. Kimura, Y. Tomioka, A. Asamitsu, and Y. Tokura, Phys. Rev. Lett. 81, 5920 (1998)

${ }^{5}$ M. Apostu, R. Suryanarayanan, A. Revcolevschi, H. Ogasawara, M. Matsukawa, M. Yoshizawa, and N. Kobayashi, Phys. Rev. B 64, 012407 (2001).

${ }^{6}$ I. Gordon, P. Wagner, V. V. Moshchalkov, Y. Bruynseraede, M. Apostu, R. Suryanarayanan, and A. Revcolevschi, Phys. Rev. B 64, 092408 (2001).

${ }^{7}$ Z. R. Liu, B. L. Gu, and X. W. Zhang, Phys. Rev. B 62, 1 (2000).

${ }^{8}$ H. Ogasawara, M. Matsukawa, S. Hatakeyama, M. Yoshizawa, M. Apostu, R. Suryanarayanan, G. Dhalenne, A. Revcolevschi, K. Ito, and N. Kobayashi, J. Phys. Soc. Jpn. 69, 1274 (2000).

${ }^{9}$ M. Kubota, H. Fujioka, K. Hirota, K. Ohyama, Y. Moritomo, H. Yoshizawa, and Y. Endoh, J. Phys. Soc. Jpn. 69, 1606 (2000).

${ }^{10}$ M. Kubota, Y. Oohara, H. Yoshizawa, H. Fujioka, K. Shimizu, K. Hirota, Y. Moritomo, and Y. Endoh, J. Phys. Soc. Jpn. 69, 1986 (2000).

${ }^{11}$ Y. Nakanishi, K. Shimomura, M. Yoshizawa, M. Apostu, R. Suryanarayanan, and A. Revcolevschi, Acta Phys. Pol. B 34, 831 (2003).

${ }^{12}$ I. Kouroudis, D. Weber, M. Yoshizawa, B. Lüthi, L. Puech, P.
Haen, J. Flouquet, G. Bruls, U. Welp, J. J. M. Franse, A. Menovsky, E. Bucher, and J. Hufnagl, Phys. Rev. Lett. 58, 820 (1987).

${ }^{13}$ P. Thalmeier, J. Magn. Magn. Mater. 76\&77, 299 (1988).

${ }^{14}$ J. R. Feller, J. B. Ketterson, D. G. Hinks, D. Dasgupta, and Bimal K. Sarma, Phys. Rev. B 62, 11538 (2000).

${ }^{15}$ B. Lüthi, J. Magn. Magn. Mater. 52, 70 (1985).

${ }^{16}$ B. Lüthi and M. Yoshizawa, J. Magn. Magn. Mater. 63\&64, 274 (1987).

${ }^{17}$ S. Nakamura, T. Goto, Y. Isikawa, S. Sakatsume, and M. Kasaya, J. Phys. Soc. Jpn. 60, 2305 (1991).

${ }^{18}$ A. Imaduddin, Y. Nakanishi, K. Shimomura, H. Kanazawa, M. Nakamura, N. Yoshimoto, and M. Yoshizawa, J. Phys. Soc. Jpn. 71, 1965 (2002).

${ }^{19}$ P. Wagner, I. Gordon, V. V. Moshchalkov, Y. Bruynseraede, M. Apostu, R. Suryanarayanan, and A. Revcolevschi, Europhys. Lett. 58, 285 (2002).

${ }^{20}$ Y. Moritomo, Y. Maruyama, T. Akimoto, and A. Nakamura, Phys. Rev. B 56, R7057 (1997).

${ }^{21}$ Joonghoe Dho, W. S. Kim, and N. H. Hur, Phys. Rev. B 65, 024404 (2001).

${ }^{22}$ J. Cao, J. T. Haraldsen, R. C. Rai, S. Brown, J. L. Musfeldt, Y. J. Wang, X. Wei, M. Apostu, R. Suryanarayanan, and A. Revcolevschi, Phys. Rev. B 74, 045113 (2006).

${ }^{23}$ F. Wang, A. Gukasov, F. Moussa, M. Hennion, M. Apostu, R. Suryanarayanan, and A. Revcolevschi, Phys. Rev. Lett. 91, 047204 (2003).

${ }^{24}$ F. Moussa, M. Hennion, F. Wang, A. Gukasov, R. Suryanarayanan, M. Apostu, and A. Revcolevschi, Phys. Rev. Lett. 93, 107202 (2004). 